Acta Crystallographica Section E

Structure Reports

Online

ISSN 1600-5368

\section{Hazel S. Wilkinson and William T. A. Harrison*}

Department of Chemistry, University of Aberdeen, Meston Walk, Aberdeen AB24 3UE, Scotland

Correspondence e-mail:

w.harrison@abdn.ac.uk

\section{Key indicators}

Single-crystal X-ray study

$T=293 \mathrm{~K}$

Mean $\sigma(\mathrm{C}-\mathrm{C})=0.003 \AA$

$R$ factor $=0.024$

$w R$ factor $=0.057$

Data-to-parameter ratio $=29.7$

For details of how these key indicators were automatically derived from the article, see http://journals.iucr.org/e.

\title{
Homopiperazinium bis(dihydrogenarsenate)
}

The crystal packing in the title compound, $\mathrm{C}_{5} \mathrm{H}_{14} \mathrm{~N}_{2}{ }^{2+}$.$2 \mathrm{H}_{2} \mathrm{AsO}_{4}{ }^{-}$, involves anion-to-anion $\mathrm{O}-\mathrm{H} \cdots \mathrm{O}$ hydrogen bonds, resulting in double chains of dihydrogenarsenate tetrahedra. The double chains are crosslinked by further $\mathrm{O}-\mathrm{H}$. . O bonds to result in a three-dimensional framework that accommodates the organic cations in large cavities. The cations interact with the framework by way of $\mathrm{N}-\mathrm{H} \cdots \mathrm{O}$ bonds.

\section{Comment}

The title compound, (I) (Fig. 1), was prepared as part of our ongoing structural studies of hydrogen-bonding interactions in protonated-amine (di)hydrogen arsenates (Wilkinson \& Harrison, 2005a,b; Todd \& Harrison, 2005). These simple organic salts show interesting packing motifs, strongly influenced by the interplay of $\mathrm{N}-\mathrm{H} \cdots \mathrm{O}$ and $\mathrm{O}-\mathrm{H} \cdots \mathrm{O}$ hydrogen bonds.<smiles>C1C[NH2+]CC[NH2+]C1</smiles>

$2\left(\mathrm{H}_{2} \mathrm{AsO}_{4}\right)^{-}$

(I)

Both the $\left(\mathrm{H}_{2} \mathrm{AsO}_{4}\right)^{-}$dihydrogenarsenate groups in (I) show their normal tetrahedral geometry [mean As-O = 1.677 (2) $\AA$ ], with the protonated As-OH vertices showing their expected lengthening relative to the unprotonated As-

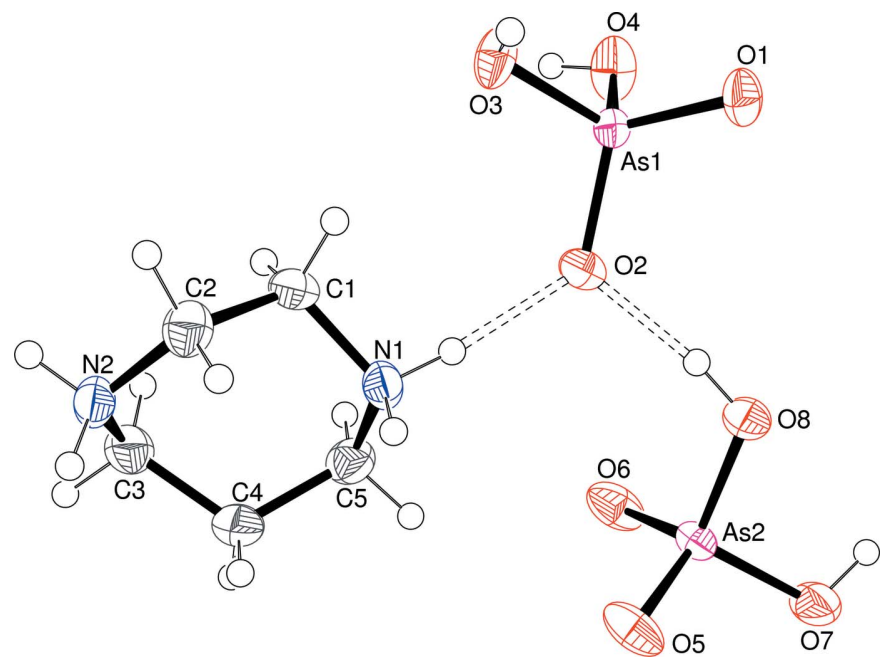

Figure 1

The asymmetric unit of (I), showing 50\% displacement ellipsoids (arbitrary spheres for $\mathrm{H}$ atoms). Hydrogen bonds are indicated by dashed lines.
Received 17 May 2006 Accepted 22 May 2006 


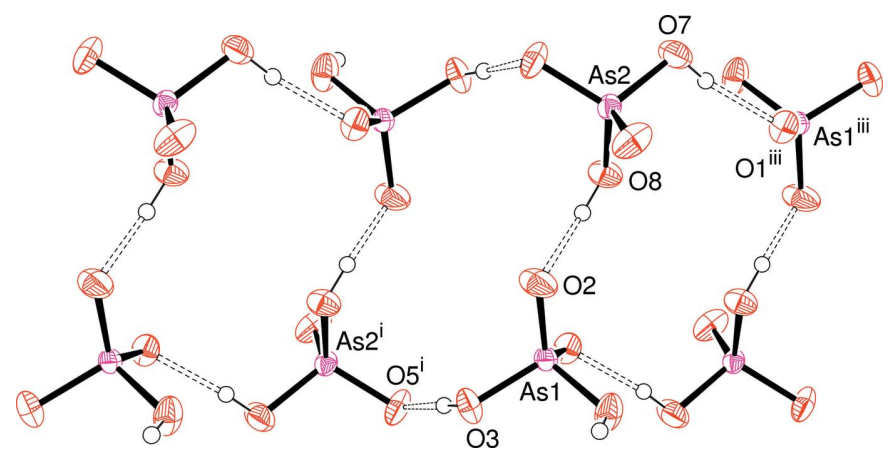

Figure 2

Detail of a hydrogen-bonded (dashed lines) dihydrogenarsenate double chain in (I). Symmetry codes are as in Table 2.

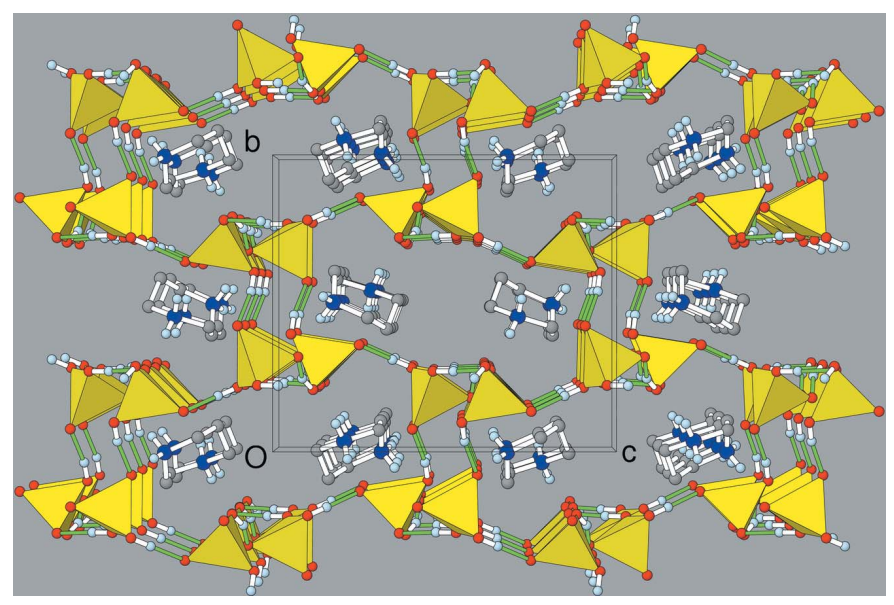

Figure 3

The packing in (I), viewed down [100], with the dihydrogenarsenate groups represented by polyhedra. Colour key: $\mathrm{H}_{2} \mathrm{AsO}_{4}{ }^{-}$groups yellow, $\mathrm{O}$ atoms red, $\mathrm{N}$ dark blue, $\mathrm{C}$ grey, $\mathrm{H}$ pale blue, $\mathrm{H} \cdots \mathrm{O}$ portions of the $\mathrm{O}-$ $\mathrm{H} \cdots \mathrm{O}$ hydrogen bonds green.

O bonds, which have formal partial double-bond character (Table 1). The homopiperazinium cation adopts a chair conformation, with atoms $\mathrm{N} 1, \mathrm{C} 1, \mathrm{C} 3$ and $\mathrm{C} 4$ almost coplanar (r.m.s. deviation from the mean plane $=0.029 \AA$ ) and atoms $\mathrm{C} 5, \mathrm{C} 2$ and $\mathrm{N} 2$ displaced from the plane by $0.667(3)$, -1.186 (3) and -1.045 (3) $\AA$, respectively.

As well as electrostatic attractions, the component species in (I) interact by means of a network of cation-to-anion $\mathrm{N}-$ $\mathrm{H} \cdots \mathrm{O}$ and anion-to-anion $\mathrm{O}-\mathrm{H} \cdots \mathrm{O}$ hydrogen bonds (Table 2). The $\left(\mathrm{H}_{2} \mathrm{AsO}_{4}\right)^{-}$units are linked into polymeric double chains (Fig. 2) propagating along [100]. Each strand of the chain consists of alternating As1- and As2-centred groups, with $\mathrm{O} 3-\mathrm{H} 1 \cdots \mathrm{O} 5^{\mathrm{i}}$ and $\mathrm{O} 7-\mathrm{H} 3 \cdots \mathrm{O} 1^{\mathrm{iii}}$ providing the hydrogen-bond links (see Table 2 for symmetry codes). The two strands are then crosslinked by the $\mathrm{O} 8-\mathrm{H} 4 \cdots \mathrm{O} 2$ interaction. The graph-set notation (Bernstein et al., 1995) for this hydrogen-bonding pattern within the double chain is an $R_{4}^{4}(16)$ loop. The As1 $\cdots \mathrm{As} 2^{\mathrm{i}}$ and $\mathrm{As} 1 \cdots \mathrm{As} 2^{\mathrm{iii}}$ intra-strand separations are 4.7032 (3) and 4.7531 (3) $\AA$, respectively, and

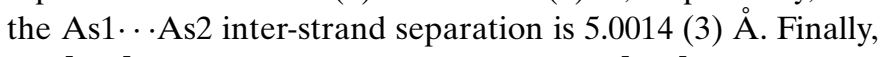
the [100] double chains are crosslinked in [001] by the O4$\mathrm{H} 2 \cdots \mathrm{O}^{\mathrm{ii}}$ bonds [with As1 $\cdots$ As $2^{\mathrm{ii}}=4.5461$ (3) $\AA$ ], to result in

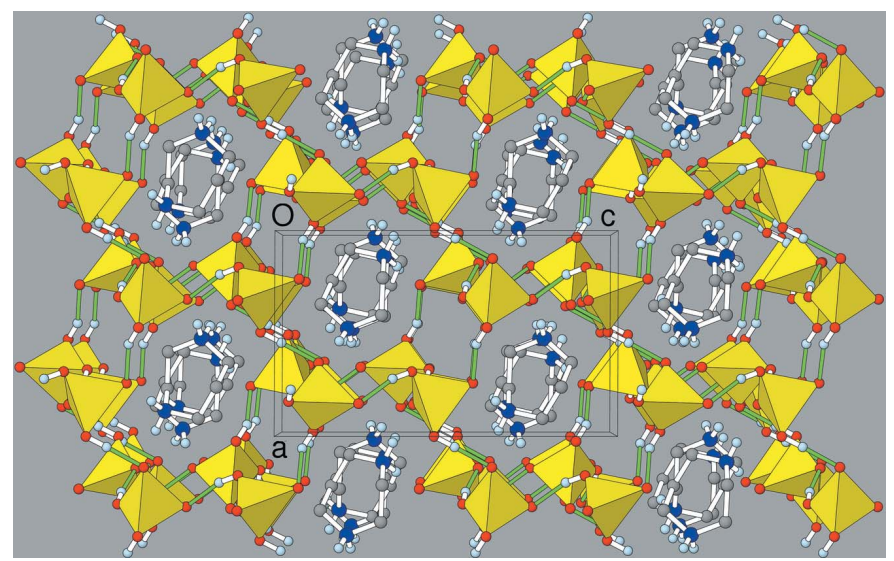

Figure 4

The packing in (I), viewed down [010]. Drawing conventions as in Fig. 3.

a very open three-dimensional network of dihydrogenarsenate groups, delimiting intersecting channels that propagate in [100] and [010] (Figs. 3 and 4). The organic cations occupy the large eight-membered ring (i.e. eight $\mathrm{H}_{2} \mathrm{AsO}_{4}$ tetrahedra) [100] channels in the framework and interact with them by way of the four $\mathrm{N}-\mathrm{H} \cdots \mathrm{O}$ bonds. It should be noted that the mean $\mathrm{H} \cdots \mathrm{O}$ contact distance for the $\mathrm{O}-\mathrm{H} \cdots \mathrm{O}$ bonds $(1.71 \AA)$ is significantly smaller than the mean $\mathrm{H} \cdots \mathrm{O}$ distance $(1.84 \AA$ ) for the $\mathrm{N}-\mathrm{H} \cdots \mathrm{O}$ bonds. The graph-set notation for the eight-membered ring loop is $R_{8}^{8}(32)$.

The situation in (I) of a hydrogen-bonded array of tetrahedral anions encompassing a network of channels occupied by organic cations is similar to that of $\alpha-\mathrm{C}_{5} \mathrm{H}_{7} \mathrm{~N}_{2} \cdot \mathrm{H}_{2} \mathrm{PO}_{4}$ $\left(\mathrm{C}_{5} \mathrm{H}_{7} \mathrm{~N}_{2}\right.$ is the 2-aminopyridinium cation; Czapla et al., 2003). In the phosphate, symmetrical $\mathrm{O} \cdots \mathrm{H} \cdots \mathrm{O}$ hydrogen bonds appear to be present at room temperature, and a paraelectricto-ferroelectric phase transition occurs on cooling below $104 \mathrm{~K}$.

\section{Experimental}

An aqueous homopiperazine solution $(10 \mathrm{ml}, 0.5 \mathrm{M})$ was added to an aqueous $\mathrm{H}_{3} \mathrm{AsO}_{4}$ solution ( $10 \mathrm{ml}, 0.5 \mathrm{M}$ ), giving a clear solution. A mass of plate- and slab-like crystals of (I) grew as the water evaporated over the course of a few days.

\section{Crystal data}

$\mathrm{C}_{5} \mathrm{H}_{14} \mathrm{~N}_{2}^{2+} \cdot 2 \mathrm{AsH}_{2} \mathrm{O}_{4}^{-}$

$M_{r}=384.05$

Monoclinic, $P 2_{1} / n$

$a=8.1495(3) \AA$

$b=11.7163$ (4) $\AA$

$c=13.5730(5) \AA$

$\beta=90.234$ (1)

$V=1295.97(8) \AA^{3}$

$Z=4$

$D_{x}=1.968 \mathrm{Mg} \mathrm{m}^{-3}$

Mo $K \alpha$ radiation

$\mu=5.19 \mathrm{~mm}^{-1}$

$T=293$ (2) K

Block cut from slab, colourless

$0.32 \times 0.19 \times 0.16 \mathrm{~mm}$

\section{Data collection}

Bruker SMART1000 CCD areadetector diffractometer $\omega$ scans

Absorption correction: multi-scan (SADABS; Bruker, 1999) $T_{\min }=0.288, T_{\max }=0.491$
14796 measured reflections 4639 independent reflections 3760 reflections with $I>2 \sigma(I)$ $R_{\text {int }}=0.025$ $\theta_{\text {max }}=32.5^{\circ}$ 


\section{Refinement}

Refinement on $F^{2}$

$R\left[F^{2}>2 \sigma\left(F^{2}\right)\right]=0.024$

$w R\left(F^{2}\right)=0.057$

$S=0.95$

4639 reflections

156 parameters

$\mathrm{H}$-atom parameters constrained $w=1 /\left[\sigma^{2}\left(F_{\mathrm{o}}{ }^{2}\right)+(0.0308 P)^{2}\right]$

$(\Delta / \sigma)_{\max }=0.001$

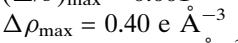

Extinction correction: SHELXL97

(Sheldrick, 1997)

Extinction coefficient: 0.0013 (3) where $P=\left(F_{\mathrm{o}}{ }^{2}+2 F_{\mathrm{c}}{ }^{2}\right) / 3$

$\Delta \rho_{\min }=-0.41{\mathrm{e} \AA^{-3}}^{-3}$

Table 1

Selected geometric parameters $\left(\AA{ }^{\circ}{ }^{\circ}\right)$.

\begin{tabular}{lrlr}
\hline As1-O1 & $1.6530(14)$ & As2-O5 & $1.6492(14)$ \\
As1-O2 & $1.6543(15)$ & As2-O6 & $1.6516(15)$ \\
As1-O4 & $1.7001(15)$ & As2-O7 & $1.6973(16)$ \\
As1-O3 & $1.7053(14)$ & As2-O8 & $1.7072(13)$ \\
& & & \\
$\mathrm{N} 1-\mathrm{C} 1-\mathrm{C} 2-\mathrm{N} 2$ & $-77.8(2)$ & $\mathrm{C} 3-\mathrm{C} 4-\mathrm{C} 5-\mathrm{N} 1$ & $65.9(3)$ \\
$\mathrm{C} 1-\mathrm{C} 2-\mathrm{N} 2-\mathrm{C} 3$ & $15.0(3)$ & $\mathrm{C} 4-\mathrm{C} 5-\mathrm{N} 1-\mathrm{C} 1$ & $-59.7(2)$ \\
$\mathrm{C} 2-\mathrm{N} 2-\mathrm{C} 3-\mathrm{C} 4$ & $55.6(3)$ & $\mathrm{C} 5-\mathrm{N} 1-\mathrm{C} 1-\mathrm{C} 2$ & $83.3(2)$ \\
$\mathrm{N} 2-\mathrm{C} 3-\mathrm{C} 4-\mathrm{C} 5$ & $-85.6(2)$ & & \\
\hline
\end{tabular}

Table 2

Hydrogen-bond geometry $\left(\AA{ }^{\circ}\right)$.

\begin{tabular}{|c|c|c|c|c|}
\hline$D-\mathrm{H} \cdots A$ & $D-\mathrm{H}$ & $\mathrm{H} \cdots A$ & $D \cdots A$ & $D-\mathrm{H} \cdots A$ \\
\hline $\mathrm{O} 3-\mathrm{H} 1 \cdots \mathrm{O}^{\mathrm{i}}$ & 0.90 & 1.65 & $2.555(2)$ & 178 \\
\hline $\mathrm{O} 4-\mathrm{H} 2 \cdots \mathrm{O}^{\mathrm{ii}}$ & 0.86 & 1.73 & $2.579(2)$ & 165 \\
\hline $\mathrm{O} 7-\mathrm{H} 3 \cdots \mathrm{O} 1^{\mathrm{iii}}$ & 0.89 & 1.76 & $2.600(2)$ & 156 \\
\hline $\mathrm{O} 8-\mathrm{H} 4 \cdots \mathrm{O} 2$ & 0.88 & 1.70 & $2.567(2)$ & 169 \\
\hline $\mathrm{N} 1-\mathrm{H} 5 \cdots \mathrm{O} 2$ & 0.90 & 1.82 & $2.716(2)$ & 174 \\
\hline $\mathrm{N} 1-\mathrm{H} 6 \cdots \mathrm{O} 1^{\mathrm{i}}$ & 0.90 & 1.91 & $2.802(2)$ & 171 \\
\hline $\mathrm{N} 2-\mathrm{H} 2 A \cdots \mathrm{O} 6^{\mathrm{iv}}$ & 0.90 & 1.80 & $2.698(2)$ & 174 \\
\hline $\mathrm{N} 2-\mathrm{H} 2 B \cdots \mathrm{O}^{\mathrm{v}}$ & 0.90 & 1.83 & $2.721(2)$ & 173 \\
\hline
\end{tabular}

\section{References}

Bernstein, J., Davis, R. E., Shimoni, L. \& Chang, N.-L. (1995). Angew. Chem. Int. Ed. Engl. 34, 1555-1573.

Bruker (1999). SMART (Version 5.624), SAINT (Version 6.02a) and $S A D A B S$. Bruker AXS Inc., Madison, Wisconsin, USA.

Czapla, Z., Dacko, S. \& Waskowska, A. (2003). J. Phys. Condens. Matter, 15, 3793-3803.

Farrugia, L. J. (1997). J. Appl. Cryst. 30, 565.

Pompetzki, M., Friese, K. \& Jansen, M. (2003). Acta Cryst. C59, i117-i119.

Sheldrick, G. M. (1990). Acta Cryst. A46, 467-473.

Sheldrick, G. M. (1997). SHELXL97. University of Göttingen, Germany.

Todd, M. J. \& Harrison, W. T. A. (2005). Acta Cryst. E61, m1024-m1026.

Wilkinson, H. S. \& Harrison, W. T. A. (2005a). Acta Cryst. E61, m1228-m1230.

Wilkinson, H. S. \& Harrison, W. T. A. (2005b). Acta Cryst. E61, m1289-m1291. 


\section{supporting information}

Acta Cryst. (2006). E62, m1397-m1399 [https://doi.org/10.1107/S1600536806018897]

\section{Homopiperazinium bis(dihydrogenarsenate)}

\section{Hazel S. Wilkinson and William T. A. Harrison}

Homopiperizinium bis(dihydrogenarsenate)

Crystal data

$\mathrm{C}_{5} \mathrm{H}_{14} \mathrm{~N}_{2}^{2+} \cdot 2 \mathrm{AsH}_{2} \mathrm{O}_{4}^{-}$

$M_{r}=384.05$

Monoclinic, $P 2_{1} / n$

Hall symbol: -P $2 \mathrm{yn}$

$a=8.1495(3) \AA$

$b=11.7163(4) \AA$

$c=13.5730(5) \AA$

$\beta=90.234(1)^{\circ}$

$V=1295.97(8) \AA^{3}$

$Z=4$

\section{Data collection}

Bruker SMART1000 CCD area-detector diffractometer

Radiation source: fine-focus sealed tube Graphite monochromator

$\omega$ scans

Absorption correction: multi-scan

(SADABS; Bruker, 1999)

$T_{\min }=0.288, T_{\max }=0.491$

Refinement

Refinement on $F^{2}$

Least-squares matrix: full

$R\left[F^{2}>2 \sigma\left(F^{2}\right)\right]=0.024$

$w R\left(F^{2}\right)=0.057$

$S=0.95$

4639 reflections

156 parameters

0 restraints

Primary atom site location: structure-invariant direct methods

Secondary atom site location: difference Fourier map
$F(000)=768$

$D_{\mathrm{x}}=1.968 \mathrm{Mg} \mathrm{m}^{-3}$

Mo $K \alpha$ radiation, $\lambda=0.71073 \AA$

Cell parameters from 6614 reflections

$\theta=2.3-32.5^{\circ}$

$\mu=5.19 \mathrm{~mm}^{-1}$

$T=293 \mathrm{~K}$

Block, colourless

$0.32 \times 0.19 \times 0.16 \mathrm{~mm}$

14796 measured reflections

4639 independent reflections

3760 reflections with $I>2 \sigma(I)$

$R_{\text {int }}=0.025$

$\theta_{\max }=32.5^{\circ}, \theta_{\min }=2.3^{\circ}$

$h=-12 \rightarrow 10$

$k=-17 \rightarrow 16$

$l=-19 \rightarrow 20$

Hydrogen site location: difmap (O-H) and geom (others)

$\mathrm{H}$-atom parameters constrained

$w=1 /\left[\sigma^{2}\left(F_{\mathrm{o}}^{2}\right)+(0.0308 P)^{2}\right]$

where $P=\left(F_{\mathrm{o}}{ }^{2}+2 F_{\mathrm{c}}{ }^{2}\right) / 3$

$(\Delta / \sigma)_{\max }=0.001$

$\Delta \rho_{\max }=0.40 \mathrm{e} \AA^{-3}$

$\Delta \rho_{\min }=-0.41$ e $\AA^{-3}$

Extinction correction: SHELXL97 (Sheldrick, 1997), $\mathrm{Fc}^{*}=\mathrm{kFc}\left[1+0.001 \mathrm{xFc}^{2} \lambda^{3} / \sin (2 \theta)\right]^{-1 / 4}$

Extinction coefficient: 0.0013 (3) 


\section{Special details}

Geometry. All e.s.d.'s (except the e.s.d. in the dihedral angle between two 1.s. planes) are estimated using the full covariance matrix. The cell e.s.d.'s are taken into account individually in the estimation of e.s.d.'s in distances, angles and torsion angles; correlations between e.s.d.'s in cell parameters are only used when they are defined by crystal symmetry. An approximate (isotropic) treatment of cell e.s.d.'s is used for estimating e.s.d.'s involving l.s. planes.

Refinement. Refinement of $F^{2}$ against ALL reflections. The weighted $R$-factor $w R$ and goodness of fit $S$ are based on $F^{2}$, conventional $R$-factors $R$ are based on $F$, with $F$ set to zero for negative $F^{2}$. The threshold expression of $F^{2}>\sigma\left(F^{2}\right)$ is used only for calculating $R$-factors (gt) etc. and is not relevant to the choice of reflections for refinement. $R$-factors based on $F^{2}$ are statistically about twice as large as those based on $F$, and $R$ - factors based on ALL data will be even larger.

Fractional atomic coordinates and isotropic or equivalent isotropic displacement parameters $\left(\hat{A}^{2}\right)$

\begin{tabular}{|c|c|c|c|c|}
\hline & $x$ & $y$ & $z$ & $U_{\text {iso }} * / U_{\text {eq }}$ \\
\hline As 1 & $0.68569(2)$ & $0.303928(15)$ & $0.034816(14)$ & $0.02193(5)$ \\
\hline $\mathrm{O} 1$ & 0.76061 (19) & $0.32062(13)$ & $-0.07725(10)$ & $0.0314(3)$ \\
\hline $\mathrm{O} 2$ & $0.6564(2)$ & $0.42518(14)$ & $0.09465(14)$ & $0.0511(5)$ \\
\hline $\mathrm{O} 3$ & 0.50652 (19) & $0.22875(14)$ & $0.03389(11)$ & $0.0341(3)$ \\
\hline H1 & 0.4560 & 0.2282 & -0.0254 & $0.041 *$ \\
\hline $\mathrm{O} 4$ & $0.8174(2)$ & $0.21845(14)$ & $0.09834(12)$ & $0.0399(4)$ \\
\hline $\mathrm{H} 2$ & 0.7830 & 0.1909 & 0.1536 & $0.048^{*}$ \\
\hline As 2 & $0.81196(2)$ & $0.702982(15)$ & $0.141776(14)$ & $0.02319(5)$ \\
\hline O5 & $0.64072(19)$ & $0.77730(14)$ & $0.13254(12)$ & $0.0383(4)$ \\
\hline O6 & $0.8354(2)$ & $0.64027(14)$ & $0.24990(10)$ & $0.0415(4)$ \\
\hline $\mathrm{O} 7$ & $0.9719(2)$ & 0.79097 (13) & $0.11673(15)$ & $0.0475(5)$ \\
\hline H3 & 1.0517 & 0.7524 & 0.0866 & $0.057^{*}$ \\
\hline O8 & $0.8236(2)$ & $0.60340(12)$ & $0.05025(10)$ & 0.0334 \\
\hline $\mathrm{H} 4$ & 0.7680 & 0.5397 & 0.0579 & $0.040 *$ \\
\hline $\mathrm{C} 1$ & 0.2683 & $0.39324(17)$ & $0.18419(15)$ & $0.0293(4)$ \\
\hline $\mathrm{H} 1 \mathrm{~A}$ & 0.2968 & 0.3474 & 0.2412 & $0.035^{*}$ \\
\hline H1B & 0.2899 & 0.3479 & 0.1259 & $0.035^{*}$ \\
\hline $\mathrm{C} 2$ & 0.0894 & $0.4205(2)$ & $0.18770(17)$ & $0.0360(5)$ \\
\hline $\mathrm{H} 2 \mathrm{C}$ & 0.0288 & 0.3538 & 0.1658 & $0.043^{*}$ \\
\hline $\mathrm{H} 2 \mathrm{D}$ & 0.0674 & 0.4813 & 0.1411 & $0.043 *$ \\
\hline N1 & $0.3756(2)$ & $0.49654(14)$ & $0.18250(13)$ & $0.0293(4)$ \\
\hline H5 & 0.4714 & 0.4778 & 0.1541 & $0.035^{*}$ \\
\hline H6 & 0.3274 & 0.5499 & 0.1445 & $0.035^{*}$ \\
\hline $\mathrm{C} 3$ & $0.1419(3)$ & $0.48886(19)$ & $0.36471(16)$ & $0.0387(5)$ \\
\hline $\mathrm{H} 3 \mathrm{~A}$ & 0.2062 & 0.4225 & 0.3832 & $0.046^{*}$ \\
\hline H3B & 0.0810 & 0.5132 & 0.4223 & $0.046^{*}$ \\
\hline $\mathrm{C} 4$ & 0.2583 & $0.58472(19)$ & $0.33405(17)$ & $0.0375(5)$ \\
\hline $\mathrm{H} 4 \mathrm{~A}$ & 0.1984 & 0.6375 & 0.2922 & $0.045^{*}$ \\
\hline H4B & 0.2915 & 0.6262 & 0.3927 & $0.045^{*}$ \\
\hline C5 & $0.4107(3)$ & $0.54720(19)$ & $0.28025(17)$ & $0.0345(5)$ \\
\hline H5A & 0.4823 & 0.6125 & 0.2718 & $0.041 *$ \\
\hline H5B & 0.4686 & 0.4916 & 0.3203 & $0.041 *$ \\
\hline N2 & $0.0242(2)$ & $0.45573(15)$ & $0.28616(15)$ & $0.0351(4)$ \\
\hline $\mathrm{H} 2 \mathrm{~A}$ & -0.0442 & 0.5150 & 0.2763 & $0.042 *$ \\
\hline $\mathrm{H} 2 \mathrm{~B}$ & -0.0368 & 0.3977 & 0.3094 & $0.042 *$ \\
\hline
\end{tabular}


Atomic displacement parameters $\left(\AA^{2}\right)$

\begin{tabular}{lllllll}
\hline & $U^{11}$ & $U^{22}$ & $U^{33}$ & $U^{12}$ & $U^{13}$ & $U^{23}$ \\
\hline As1 & $0.02024(9)$ & $0.02205(9)$ & $0.02354(9)$ & $-0.00063(7)$ & $0.00305(7)$ & $0.00010(7)$ \\
O1 & $0.0299(7)$ & $0.0391(8)$ & $0.0253(7)$ & $0.0027(6)$ & $0.0061(6)$ & $0.0103(6)$ \\
O2 & $0.0463(10)$ & $0.0324(8)$ & $0.0749(13)$ & $-0.0089(7)$ & $0.0309(9)$ & $-0.0224(8)$ \\
O3 & $0.0252(7)$ & $0.0476(9)$ & $0.0295(7)$ & $-0.0123(7)$ & $-0.0023(6)$ & $0.0063(7)$ \\
O4 & $0.0291(8)$ & $0.0560(10)$ & $0.0346(8)$ & $0.0050(7)$ & $-0.0018(7)$ & $0.0176(7)$ \\
As2 & $0.02066(9)$ & $0.02539(9)$ & $0.02351(9)$ & $0.00275(7)$ & $-0.00057(7)$ & $-0.00614(7)$ \\
O5 & $0.0277(8)$ & $0.0512(9)$ & $0.0360(8)$ & $0.0179(7)$ & $-0.0075(6)$ & $-0.0145(7)$ \\
O6 & $0.0559(11)$ & $0.0449(9)$ & $0.0237(7)$ & $0.0201(8)$ & $-0.0018(7)$ & $-0.0036(6)$ \\
O7 & $0.0350(9)$ & $0.0323(9)$ & $0.0754(14)$ & $-0.0087(7)$ & $0.0180(9)$ & $-0.0190(8)$ \\
O8 & $0.0436(9)$ & $0.0294(7)$ & $0.0271(7)$ & $-0.0041(7)$ & $0.0047(7)$ & $-0.0092(6)$ \\
C1 & $0.0353(11)$ & $0.0238(9)$ & $0.0287(9)$ & $0.0041(8)$ & $0.0010(8)$ & $-0.0031(8)$ \\
C2 & $0.0287(11)$ & $0.0394(12)$ & $0.0400(12)$ & $-0.0041(9)$ & $-0.0013(9)$ & $-0.0004(9)$ \\
N1 & $0.0259(8)$ & $0.0301(9)$ & $0.0322(9)$ & $0.0045(7)$ & $0.0074(7)$ & $0.0058(7)$ \\
C3 & $0.0494(14)$ & $0.0342(11)$ & $0.0326(11)$ & $0.0016(10)$ & $0.0135(10)$ & $0.0013(9)$ \\
C4 & $0.0451(13)$ & $0.0277(10)$ & $0.0396(12)$ & $-0.0009(9)$ & $0.0066(10)$ & $-0.0077(9)$ \\
C5 & $0.0298(11)$ & $0.0296(11)$ & $0.0441(12)$ & $-0.0033(8)$ & $-0.0020(9)$ & $-0.0014(9)$ \\
N2 & $0.0273(9)$ & $0.0292(9)$ & $0.0488(11)$ & $0.0024(7)$ & $0.0115(8)$ & $0.0103(8)$ \\
& & & & & &
\end{tabular}

Geometric parameters $(\AA, \stackrel{\circ}{)})$

\begin{tabular}{|c|c|c|c|}
\hline As1-O1 & $1.6530(14)$ & $\mathrm{C} 2-\mathrm{H} 2 \mathrm{C}$ & 0.9700 \\
\hline $\mathrm{As} 1-\mathrm{O} 2$ & $1.6543(15)$ & $\mathrm{C} 2-\mathrm{H} 2 \mathrm{D}$ & 0.9700 \\
\hline As1-O4 & $1.7001(15)$ & $\mathrm{N} 1-\mathrm{C} 5$ & $1.480(3)$ \\
\hline $\mathrm{As} 1-\mathrm{O} 3$ & $1.7053(14)$ & $\mathrm{N} 1-\mathrm{H} 5$ & 0.9000 \\
\hline $\mathrm{O} 3-\mathrm{H} 1$ & 0.9028 & $\mathrm{~N} 1-\mathrm{H} 6$ & 0.9000 \\
\hline $\mathrm{O} 4-\mathrm{H} 2$ & 0.8642 & $\mathrm{C} 3-\mathrm{N} 2$ & $1.483(3)$ \\
\hline As $2-\mathrm{O} 5$ & $1.6492(14)$ & $\mathrm{C} 3-\mathrm{C} 4$ & $1.529(3)$ \\
\hline As $2-\mathrm{O} 6$ & $1.6516(15)$ & $\mathrm{C} 3-\mathrm{H} 3 \mathrm{~A}$ & 0.9700 \\
\hline As $2-07$ & $1.6973(16)$ & $\mathrm{C} 3-\mathrm{H} 3 \mathrm{~B}$ & 0.9700 \\
\hline As2-O8 & $1.7072(13)$ & $\mathrm{C} 4-\mathrm{C} 5$ & $1.509(3)$ \\
\hline $\mathrm{O} 7-\mathrm{H} 3$ & 0.8921 & $\mathrm{C} 4-\mathrm{H} 4 \mathrm{~A}$ & 0.9700 \\
\hline $\mathrm{O} 8-\mathrm{H} 4$ & 0.8799 & $\mathrm{C} 4-\mathrm{H} 4 \mathrm{~B}$ & 0.9700 \\
\hline $\mathrm{C} 1-\mathrm{N} 1$ & $1.493(3)$ & $\mathrm{C} 5-\mathrm{H} 5 \mathrm{~A}$ & 0.9700 \\
\hline $\mathrm{C} 1-\mathrm{C} 2$ & $1.494(3)$ & $\mathrm{C} 5-\mathrm{H} 5 \mathrm{~B}$ & 0.9700 \\
\hline $\mathrm{C} 1-\mathrm{H} 1 \mathrm{~A}$ & 0.9700 & $\mathrm{~N} 2-\mathrm{H} 2 \mathrm{~A}$ & 0.9000 \\
\hline $\mathrm{C} 1-\mathrm{H} 1 \mathrm{~B}$ & 0.9700 & $\mathrm{~N} 2-\mathrm{H} 2 \mathrm{~B}$ & 0.9000 \\
\hline $\mathrm{C} 2-\mathrm{N} 2$ & $1.498(3)$ & & \\
\hline $\mathrm{O} 1-\mathrm{As} 1-\mathrm{O} 2$ & $113.88(8)$ & $\mathrm{C} 5-\mathrm{N} 1-\mathrm{H} 5$ & 108.5 \\
\hline $\mathrm{O} 1-\mathrm{As} 1-\mathrm{O} 4$ & $107.56(8)$ & $\mathrm{C} 1-\mathrm{N} 1-\mathrm{H} 5$ & 108.5 \\
\hline $\mathrm{O} 2-\mathrm{As} 1-\mathrm{O} 4$ & $110.43(9)$ & $\mathrm{C} 5-\mathrm{N} 1-\mathrm{H} 6$ & 108.5 \\
\hline $\mathrm{O} 1-\mathrm{As} 1-\mathrm{O} 3$ & $111.95(8)$ & $\mathrm{C} 1-\mathrm{N} 1-\mathrm{H} 6$ & 108.5 \\
\hline $\mathrm{O} 2-\mathrm{As} 1-\mathrm{O} 3$ & $108.77(8)$ & $\mathrm{H} 5-\mathrm{N} 1-\mathrm{H} 6$ & 107.5 \\
\hline $\mathrm{O} 4-\mathrm{As} 1-\mathrm{O} 3$ & $103.78(8)$ & $\mathrm{N} 2-\mathrm{C} 3-\mathrm{C} 4$ & $113.39(18)$ \\
\hline $\mathrm{As} 1-\mathrm{O} 3-\mathrm{H} 1$ & 113.4 & $\mathrm{~N} 2-\mathrm{C} 3-\mathrm{H} 3 \mathrm{~A}$ & 108.9 \\
\hline
\end{tabular}




$\begin{array}{llll}\text { As1-O4-H2 } & 117.0 & \mathrm{C} 4-\mathrm{C} 3-\mathrm{H} 3 \mathrm{~A} & 108.9 \\ \mathrm{O} 5-\mathrm{As} 2-\mathrm{O} 6 & 113.40(8) & \mathrm{N} 2-\mathrm{C} 3-\mathrm{H} 3 \mathrm{~B} & 108.9 \\ \mathrm{O} 5-\mathrm{As} 2-\mathrm{O} 7 & 108.32(9) & \mathrm{C} 4-\mathrm{C} 3-\mathrm{H} 3 \mathrm{~B} & 108.9 \\ \mathrm{O} 6-\mathrm{As} 2-\mathrm{O} 7 & 111.23(10) & \mathrm{H} 3 \mathrm{~A}-\mathrm{C} 3-\mathrm{H} 3 \mathrm{~B} & 107.7 \\ \mathrm{O} 5-\mathrm{As} 2-\mathrm{O} 8 & 110.81(8) & \mathrm{C} 5-\mathrm{C} 4-\mathrm{C} 3 & 115.50(18) \\ \mathrm{O} 6-\mathrm{As} 2-\mathrm{O} 8 & 109.63(7) & \mathrm{C} 5-\mathrm{C} 4-\mathrm{H} 4 \mathrm{~A} & 108.4 \\ \mathrm{O} 7-\mathrm{As} 2-\mathrm{O} 8 & 102.95(8) & \mathrm{C} 3-\mathrm{C} 4-\mathrm{H} 4 \mathrm{~A} & 108.4 \\ \mathrm{As} 2-\mathrm{O} 7-\mathrm{H} 3 & 110.2 & \mathrm{C} 5-\mathrm{C} 4-\mathrm{H} 4 \mathrm{~B} & 108.4 \\ \mathrm{As} 2-\mathrm{O} 8-\mathrm{H} 4 & 117.6 & \mathrm{C} 3-\mathrm{C} 4-\mathrm{H} 4 \mathrm{~B} & 107.5 \\ \mathrm{~N} 1-\mathrm{C} 1-\mathrm{C} 2 & 113.53(17) & \mathrm{H} 4 \mathrm{~A}-\mathrm{C} 4-\mathrm{H} 4 \mathrm{~B} & 113.23(18) \\ \mathrm{N} 1-\mathrm{C} 1-\mathrm{H} 1 \mathrm{~A} & 108.9 & \mathrm{~N} 1-\mathrm{C} 5-\mathrm{C} 4 & 108.9 \\ \mathrm{C} 2-\mathrm{C} 1-\mathrm{H} 1 \mathrm{~A} & 108.9 & \mathrm{~N} 1-\mathrm{C} 5-\mathrm{H} 5 \mathrm{~A} & 108.9 \\ \mathrm{~N} 1-\mathrm{C} 1-\mathrm{H} 1 \mathrm{~B} & 108.9 & \mathrm{C} 4-\mathrm{C} 5-\mathrm{H} 5 \mathrm{~A} & 108.9 \\ \mathrm{C} 2-\mathrm{C} 1-\mathrm{H} 1 \mathrm{~B} & 108.9 & \mathrm{~N} 1-\mathrm{C} 5-\mathrm{H} 5 \mathrm{~B} & 108.9 \\ \mathrm{H} 1 \mathrm{~A}-\mathrm{C} 1-\mathrm{H} 1 \mathrm{~B} & 107.7 & \mathrm{C} 4-\mathrm{C} 5-\mathrm{H} 5 \mathrm{~B} & 107.7 \\ \mathrm{C} 1-\mathrm{C} 2-\mathrm{N} 2 & 115.93(19) & \mathrm{H} 5 \mathrm{~A}-\mathrm{C} 5-\mathrm{H} 5 \mathrm{~B} & 118.85(17) \\ \mathrm{C} 1-\mathrm{C} 2-\mathrm{H} 2 \mathrm{C} & 108.3 & \mathrm{C} 3-\mathrm{N} 2-\mathrm{C} 2 & 107.6 \\ \mathrm{~N} 2-\mathrm{C} 2-\mathrm{H} 2 \mathrm{C} & 108.3 & \mathrm{C} 3-\mathrm{N} 2-\mathrm{H} 2 \mathrm{~A} & 107.6 \\ \mathrm{C} 1-\mathrm{C} 2-\mathrm{H} 2 \mathrm{D} & 108.3 & \mathrm{C} 2-\mathrm{N} 2-\mathrm{H} 2 \mathrm{~A} & 107.6 \\ \mathrm{~N} 2-\mathrm{C} 2-\mathrm{H} 2 \mathrm{D} & 108.3 & \mathrm{C} 3-\mathrm{N} 2-\mathrm{H} 2 \mathrm{~B} & 107.6 \\ \mathrm{H} 2 \mathrm{C}-\mathrm{C} 2-\mathrm{H} 2 \mathrm{D} & 107.4 & \mathrm{C} 2-\mathrm{N} 2-\mathrm{H} 2 \mathrm{~B} & 107.0 \\ \mathrm{C} 5-\mathrm{N} 1-\mathrm{C} 1 & 114.97(16) & \mathrm{H} 2 \mathrm{~A}-\mathrm{N} 2-\mathrm{H} 2 \mathrm{~B} & 65.9(3) \\ & & & -59.7(2) \\ \mathrm{N} 1-\mathrm{C} 1-\mathrm{C} 2-\mathrm{N} 2 & -77.8(2) & \mathrm{C} 3-\mathrm{C} 4-\mathrm{C} 5-\mathrm{N} 1 & 83.3(2) \\ \mathrm{C} 1-\mathrm{C} 2-\mathrm{N} 2-\mathrm{C} 3 & 15.0(3) & \mathrm{C} 4-\mathrm{C} 5-\mathrm{N} 1-\mathrm{C} 1 & \\ \mathrm{C} 2-\mathrm{N} 2-\mathrm{C} 3-\mathrm{C} 4 & 55.6(3) & \mathrm{C} 5-\mathrm{N} 1-\mathrm{C} 1-\mathrm{C} 2 & \\ \mathrm{~N} 2-\mathrm{C} 3-\mathrm{C} 4-\mathrm{C} 5 & -85.6(2) & & \end{array}$

Hydrogen-bond geometry $\left(\AA,{ }^{\circ}\right)$

\begin{tabular}{lllll}
\hline$D-\mathrm{H} \cdots A$ & $D-\mathrm{H}$ & $\mathrm{H} \cdots A$ & $D \cdots A$ & $D-\mathrm{H} \cdots A$ \\
\hline $\mathrm{O} 3-\mathrm{H} 1 \cdots \mathrm{O}^{\mathrm{i}}$ & 0.90 & 1.65 & $2.555(2)$ & 178 \\
$\mathrm{O} 4-\mathrm{H} 2 \cdots 6^{\mathrm{ii}}$ & 0.86 & 1.73 & $2.579(2)$ & 165 \\
$\mathrm{O} 7-\mathrm{H} 3 \cdots \mathrm{O} 1^{\mathrm{iii}}$ & 0.89 & 1.76 & $2.600(2)$ & 156 \\
$\mathrm{O} 8-\mathrm{H} 4 \cdots \mathrm{O} 2$ & 0.88 & 1.70 & $2.567(2)$ & 169 \\
$\mathrm{~N} 1-\mathrm{H} 5 \cdots \mathrm{O} 2$ & 0.90 & 1.82 & $2.716(2)$ & 174 \\
$\mathrm{~N} 1-\mathrm{H} 6 \cdots{ }^{\mathrm{i}}$ & 0.90 & 1.91 & $2.802(2)$ & 171 \\
$\mathrm{~N} 2-\mathrm{H} 2 A \cdots \mathrm{O}^{\text {iv }}$ & 0.90 & 1.80 & $2.698(2)$ & 174 \\
$\mathrm{~N} 2-\mathrm{H} 2 B \cdots 5^{\mathrm{v}}$ & 0.90 & 1.83 & $2.721(2)$ & 173
\end{tabular}

Symmetry codes: (i) $-x+1,-y+1,-z$; (ii) $-x+3 / 2, y-1 / 2,-z+1 / 2$; (iii) $-x+2,-y+1,-z$; (iv) $x-1, y, z$; (v) $-x+1 / 2, y-1 / 2,-z+1 / 2$. 